YITP-SB-02-75

SLAC-PUB-9606

December 2002

\title{
An Elementary Aharonov-Bohm System in Three Space Dimensions: Quantum Attraction With No Classical Force*
}

\author{
Alfred Scharff Goldhaber ${ }^{a, b, \dagger}$ and Ryan Requist ${ }^{a, \ddagger}$ \\ ${ }^{a}$ C. N. Yang Institute for Theoretical Physics and \\ Department of Physics and Astronomy \\ State University of New York \\ Stony Brook, NY 11794-3840 \\ and \\ ${ }^{b}$ Stanford Linear Accelerator Center, Menlo Park, CA 94025
}

\begin{abstract}
As a consequence of the Aharonov-Bohm effect, there is a quantum-induced attraction between a charged particle and a rigid, impenetrable hoop made from an arbitrarily thin tube containing a superconductor quantum of magnetic flux. This is remarkable because in classical physics there is no force between the two objects, and quantum-mechanical effects (associated with uncertainty principle energy) generally are repulsive rather than attractive. For an incident spinless charged particle in a $P$ wave (in a configuration with total angular momentum zero) we verify a resonance just above threshold using the Kohn variational principle in its S-matrix form. Even if optimistic choices of parameters describing a model system with these properties turned out to be feasible, the temperature required to observe the resonance would be far lower than has yet been attained in the laboratory.
\end{abstract}

PACS numbers: 03.65.Vf, 03.65.Nk, 31.15 Pf

*Work supported in part by the Department of Energy under contract number DE-AC03-76SF00515 and by the National Science Foundation under Grant PHY0140192 .

† goldhab@insti.physics.sunysb.edu

${ }^{\ddagger}$ rrequist@grad.physics.sunysb.edu 


\section{Introduction}

The Aharonov-Bohm effect [1] is among the most striking of quantum phenomena. While the value of the vector potential at a point has no observable significance, and the value of the field strength in a region accessible to an electron is not completely adequate to describe the influence of the electromagnetic field, the exponential line integral around every accessible closed circuit

$$
\exp \left(i \frac{e}{\hbar} \oint d \vec{r} \cdot \vec{A}\right)
$$

(a manifestly gauge invariant form) indeed is exactly the right quantity to capture fully all electromagnetic effects [2]. The phase associated with the effect is manifested through displacement in the interference fringes observed in a two-slit electron diffraction experiment, depending on the magnetic flux enclosed between the separated beams. One also finds perturbations of the energy eigenvalues of a charged particle bound in a region surrounding an impenetrable tube of magnetic flux, again depending on the magnitude of that flux, or rather the amount by which it differs from an integer number of flux quanta $h / e$. The AB effect may be understood as a topological effect (a holonomy) arising from a cyclic variation in the projective Hilbert space [3, 4, 5]. The two-body problem of charge interacting with flux has been studied in two dimensions. The aim of this paper is to investigate the corresponding two-body $\mathrm{AB}$ problem in three dimensions, where the simplest system, possessing the fewest degrees of freedom, consists of a charged particle interacting with a rigid circular hoop of magnetic flux. Of finite length, the (arbitrarily thin) flux tube has finite mass, and so this system presents a well-defined two-body problem, in which the degrees of freedom are the relative translational motion between charge and hoop, and the rotational motion of the direction normal to the plane of the hoop. The extra rotational degree of freedom raises the possibility of an attraction or even a bound state between charge and hoop.

In a quantum setting, bound states appear which have no classical analogue. An example in two space dimensions [6] is a state localized at the intersection of two channels whose walls form the boundaries of forbidden regions. A particle state in the vicinity of the junction has a greater spatial uncertainty and hence lower mean square momentum than a freely propagating state in either individual channel. Because a particle in this state lacks the energy to propagate indefinitely down either channel, its wave function decays exponentially with distance from the intersection. One can envision other circumstances in which reduced uncertainty-principle energy gives rise to binding for quantum systems. In fact, the same phenomenon can be found in solutions of wave equations even for macroscopic systems. Exner and Seba [7] and Goldstone and Jaffe [8] studied the bound state present whenever a waveguide has 
a bend. The analysis applies to waves in any non-dispersive medium satisfying the wave equation subject to Dirichlet boundary conditions.

The three-dimensional system we consider has no forbidden regions or classical forces, yet the particle experiences an attraction. The lack of classical forces is based on the assumption that the flux is contained in a tube of negligible radius. For the same reason, even in the quantum context one need not assume an explicit repulsive force to keep the spinless charged particle from penetrating the flux, because already in their original work Aharonov and Bohm [1] showed that the wave function automatically vanishes at the location of the flux. This fact may be viewed as an example of the much more typical influence of quantum corrections to a classical picture, namely repulsion.

If we track the phase of the wave function along a path that links the hoop (using a singular gauge in which the vector potential vanishes almost everywhere), then as mentioned already the $\mathrm{AB}$ effect generates a discontinuity or phase jump

$$
\Delta \phi=\frac{e}{\hbar} \oint d \vec{r} \cdot \vec{A}(\vec{r})=\frac{e}{\hbar} \Phi_{B}
$$

When the hoop contains a superconductor quantum of flux, $\Phi_{B}=h / 2 e$, the phase discontinuity is $\pi$. This implies the existence of a surface bounded by the hoop on which the wave function vanishes, and across which the wave function changes sign. From symmetry arguments, at zero kinetic energy of the particle either of the two natural choices for this surface is coplanar with the hoop. One surface is the portion of the plane inside the hoop and corresponds to odd partial waves inside and even partial waves outside a sphere for which the hoop forms an equator. The other is in the region outside the hoop and leads to reverse parity. Here 'parity' refers to factors of $\cos \theta$ in the wave function, where $\theta$ is the polar angle of the particle with respect to a normal to the hoop plane erected over the center of the hoop. To conserve angular momentum the hoop must change its rotational state when the particle crosses the hoop radius, $r=R$. Suppose that initially the particle and hoop together have zero total angular momentum. In the scattering of a $P$ wave the particle may enter a state of zero orbital angular momentum inside, where the absence of the centrifugal barrier corresponds to an attraction. A generic profile of the resulting effective potential is shown in Figure 1 .

In Sec 2, we examine the qualitative features of the interaction. A variational calculation gives an upper bound, incidentally above threshold, for the eigenenergy of a hypothetical bound state. Sec 2.2 presents a simple proof precluding the existence of a bound state but anticipating a resonance. Its properties are described in Sec 3 , where the S-matrix version of the Kohn variational principle is used to determine the partial wave amplitudes. In Sec 4 we present a short discussion of the feasibility of detecting the resonance for a system consisting of a carbon nanotube bent into a 


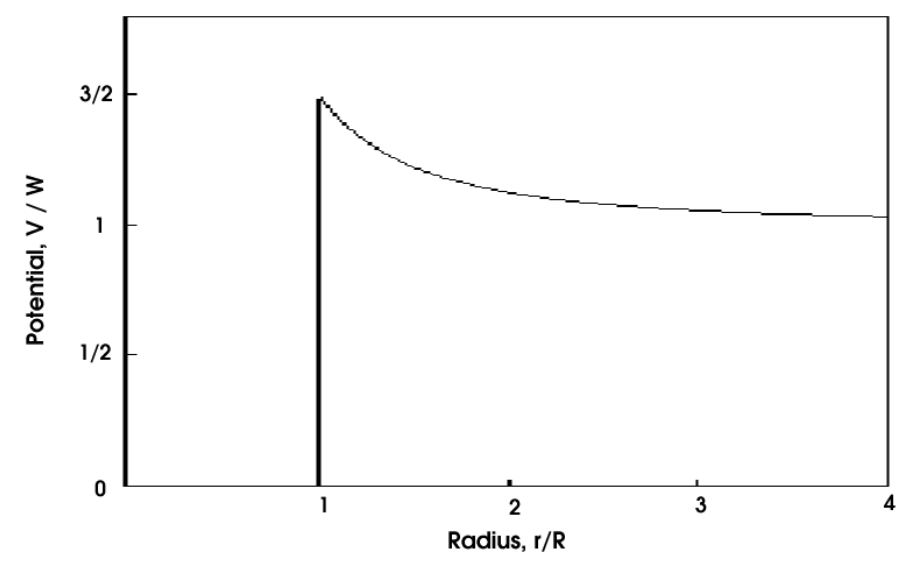

Figure 1: The effective potential in units of the threshold energy for odd partial waves outside, $S$ wave inside. The potential has the standard centrifugal form, but with a constant offset representing the kinetic energy of the spinning hoop.

circle, interacting with a heavy charged particle. Temperatures required for detection would be far below values currently attainable in the laboratory.

\section{The Absence of a Bound State}

We first investigate the possibility of a bound state. Consider a state with zero total angular momentum and even parity in the interior region. The highest possible energy for a bound state is the energy of the lowest lying exterior channel - the $P$ state - at infinite separation of the particle and hoop. As the orbital energy tends to zero in this limit, the threshold is simply the energy of the hoop with one quantum of angular momentum, $W \equiv 2 \hbar^{2} / 2 I\left(I=\frac{1}{2} m R^{2}\right)$. If the hoop is static the connection between interior and exterior partial waves is straightforward. The neglect of hoop rotation for the purpose of matching (we do not neglect this rotation as a contributor to the energy) will be referred to as the static hoop matching conditions (Eq. 31). The crux of the problem is the complication arising from the dynamics of the hoop rotation. An approach which we shall not pursue here would be transforming to the rotating instantaneous rest frame of the hoop, clearly a noninertial frame leading to coriolis forces which would complicate the analysis.

We can understand the qualitative features of the interaction by looking at limiting cases. Let us introduce two characteristic frequencies. The transit frequency $\nu_{T}$ is defined as the reciprocal mean passage time of a particle across the diameter of a non-interacting hoop, and the rotational frequency $\nu_{R}$ is associated with the hoop 
rotation:

$$
\begin{aligned}
& \nu_{T}=\frac{v}{2 R}=\frac{\sqrt{2 E / m}}{2 R} \\
& \nu_{R}=\frac{\delta E_{\text {hoop }}}{h}=\frac{1}{h} \frac{\delta\left(L^{2}\right)}{2 I}=\frac{\hbar}{2 \pi} \frac{l^{\prime}\left(l^{\prime}+1\right)-l(l+1)}{m R^{2}} .
\end{aligned}
$$

The regimes of fast and slow rotational motion are realized when the ratio $\nu_{R} / \nu_{T}$ is respectively large or small, in which case either the relative motion or the rotational motion may be treated as an adiabatic variable. In the following two sections, we examine the qualitative features of the interaction in these two regimes.

\subsection{Variational Upper Bound}

We address the issue of a bound state by seeking an upper bound to the eigenenergy in the regime of slow rotational motion. We allow the greatest freedom for the existence of a bound state by assuming infinite mass for the charged particle, thereby maximizing the reduced mass and minimizing the translational kinetic energy. In the center of mass frame the reduced particle moves relative to a hoop with fixed center. For $\nu_{R} / \nu_{T} \rightarrow 0$, the condition for continuity of probability density at the boundary $r=R$ becomes

$$
\begin{gathered}
\int_{-1}^{1} d \mu\left|\Psi_{\text {in }}(R, \mu)-\Psi_{\text {out }}(R, \mu)\right|^{2}=0, \text { or } \\
\int_{-1}^{1} d \mu\left|\sum_{0, \text { even } n} c_{n} f_{n}\left(k_{n} R\right) P_{n}(\mu)-\epsilon(\mu) \sum_{\text {odd } l} c_{l} f_{l}\left(k_{l} R\right) P_{l}(\mu)\right|^{2}=0
\end{gathered}
$$

A Legendre polynomial $\left(P_{n}(\mu)\right)$ of odd order and another of even order are orthogonal simply because the former is odd on the interval $\mu:-1 \rightarrow 1$, while the latter is even. The argument of the polynomial is $\mu=\cos (\hat{r} \cdot \hat{n})$, where $\hat{n}$ is the unit normal along the symmetry axis of the hoop. In the present model, paths traversing above and below the hoop circumference give a relative sign, which is represented in the equation by the function $\epsilon(\mu) \equiv \mu /|\mu|$. In forming the projection of states outside the hoop radius onto states inside the hoop radius, those of different parity are not decoupled, while states of equal parity are decoupled. The static hoop matching conditions involve taking as the nominal amplitude of each exterior partial wave, $c_{l}$, its projection onto the interior wave function. These conditions are accurate in the limit of high energy with fixed maximum value for the orbital angular momentum. The candidate wave function in the interior region is taken to be a constant, because at low energies the 
$S$ wave, expected to give the dominant contribution, has a relatively weak radial dependence and is isotropic. The exterior trial function is composed of odd partial waves with Laplacian radial dependence, $f_{l}\left(k_{l} r\right)=1 /\left(k_{l} r\right)^{l+1}$, and Legendre angular functions, $P_{l}(\cos \mu)$. A simple estimate shows that the series for the expected energy,

$$
\langle\Psi|H| \Psi\rangle=\int d^{3} r \sum_{l}\left[\frac{\hbar^{2}}{2 m_{\mu}}\left|\nabla \psi_{l}\right|^{2}+\frac{\hbar^{2} l(l+1)}{2 m_{\mu} r^{2}}\left|\psi_{l}\right|^{2}+\frac{\hbar^{2} l(l+1)}{m_{H} R^{2}}\left|\psi_{l}\right|^{2}\right]
$$

is divergent. In the present case the sum over $l$ runs over zero (inside) and the odd integers (outside). The parity of the partial wave, $\psi_{l}$, determines the region of integration, i.e., the volume either inside or outside the hoop radius. The energy has contributions from the radial and orbital motion of the reduced particle with mass $m_{\mu}$ and the rotation of the hoop with mass $m_{H}$. As the charged particle is artificially given an infinite mass, we have $m_{\mu}=m_{H}$. From the coupling and the angular factors alone, the outside functions have asymptotic normalization going as $1 / l^{2}$ and with the radial integrals, $1 / l^{3}$. The energy, containing the factor $l(l+1)$, goes as $1 / l$, resulting in a logarithmically divergent sum.

We may obtain a more realistic bound by optimizing the trial function with the appropriate though less tractable Bessel functions. Opening a finite number of interior channels, $N / 2+1$, and a (substantially larger) number of exterior channels, $L / 2+1 / 2$, the coefficients of the interior partial waves become free parameters under the static hoop connection rules. Here $N$ and $L$ label the cutoffs for the truncation of the series in Eq. 4. The expectation value of the energy is minimized with respect to the interior amplitudes. Again, for a given value of $N$ the energy is logarithmically divergent in $L$. However, the divergence is damped in the limit $N \rightarrow \infty$, and we find a convergence of the extrapolated energy at approximately $14 \%$ above threshold. In general the energy has a weak dependence on $L$ or $N$. The trend hints that the influence of a partial wave diminishes rapidly with its order - an idea that will reemerge when we study the resonance. At this point, one cannot make a definitive statement regarding binding, though the results suggest it is unlikely.

A reader might well ask why only Legendre polynomials and not all spherical harmonics are used to describe the orbital motion. The ideal hoop has zero projection of spin about its axis of symmetry, and hence for total angular momentum zero also the orbital angular momentum about the symmetry axis must vanish. That is exactly the criterion for keeping just Legendre polynomials.

\subsection{Lower Bound}

In view of the previous result, we suspect a bound state might be described better with the assumption of fast rotational motion. Let us look at this other limit and 
in the process rule out the possibility of a bound state. Clearly there is uncertaintyprinciple energy associated with the confinement of the particle to a finite region near the hoop. The effective potential encountered by the particle in an exterior channel has the generic form shown in Figure1. The particle experiences a centrifugal barrier, going as $1 / r^{2}$, associated with its angular momentum. The constant offset, defining the threshold energy for a partial wave, is the rotational energy of the hoop.

Consider a state with exclusively internal $S$ and external $P$ partial waves. The rotation of the hoop is not an issue, because the amplitude connecting the $S$ and $P$ waves and the phase shift are entirely determined by the continuity conditions at the boundary $r=R$. A necessary condition for continuity of wave function amplitude and radial gradient is continuity of logarithmic derivative. At energies up to the threshold, the logarithmic derivatives remain discontinuous. For the $S$ wave inside, the logarithmic derivative at threshold is $(2 / R) \cot (2)-1 / R$. For the $P$ wave outside, it is $-2 / R$, giving a ratio between inside and outside of 0.958 . This indicates slightly too much radial kinetic energy for binding.

One can go further and consider the inclusion of higher partial waves in the trial wave function. However, the energy of such a function will not be an extremum, as it obviously is lowered by reducing the wave function to exclusively the lowest allowed partial wave $(S$ or $P)$. Therefore, we conclude that the Schrödinger equation subject to the continuity conditions has no solution below threshold, i.e., no bound state.

\section{Resonance}

\subsection{The $S / P$ coupling}

The attraction between the particle and hoop guarantees the existence of a resonance. In the previous section, we found the ratio of the interior to exterior logarithmic derivatives to be approximately 0.958 at the threshold energy. This ratio, nearly unity, strongly suggests a resonance slightly above threshold. Suppose that were so. Then the ratio of frequencies is $\nu_{R} / \nu_{T} \approx \mathcal{O}(1)$. In this range of energy, neither the limit of fast rotational nor fast translational motion is strictly valid. The $S$ and $P$ waves (the only classically allowed channels for the radial motion) are expected to hold the largest share of the probability, and exclusion of higher partial waves should be a suitable first approximation. We can find a more compelling argument for neglecting higher partial waves. The S-matrix version [9] of the Kohn variational principle [10] provides us with a criterion for estimating the contribution of higher partial waves. We remark that the existence of a resonance implies a pole in the S-matrix off the real axis in energy. We confine our scope to energies below the threshold of the $F$ $(l=3)$ channel. Therefore we are only considering single channel scattering, though 
the case of multichannel scattering is an interesting one for possible future study. The S-matrix now is simply a $U(1)$ matrix in which the phase shift is encoded, $S=e^{2 i \delta}$. The variational approximation to the S-matrix is

$$
S=\operatorname{ext}\left[\tilde{S}+\frac{i}{\hbar}\langle\tilde{\psi}|H-E| \tilde{\psi}\rangle\right]
$$

The extremum ("ext") is taken with respect to all free parameters $c_{j}$ of the trial function $\tilde{\psi}$. As we choose a trial function with linear superpositions of the energy eigenstates (obeying the continuity conditions on density and current at $r=R$ ), the second term in Eq. (5) vanishes identically, and the principle takes on a remarkably simple form. In essence, this is a because the AB effect acts in a singular manner, i.e. we have everywhere the free particle Hamiltonian, except at $r=R$ (where the continuity conditions assure $\langle\tilde{\psi}|H-E| \tilde{\psi}\rangle=0)$ :

$\tilde{\psi}(r, \mu)= \begin{cases}h_{1}^{(2)}\left(k_{1} r\right) P_{1}(\mu)+S h_{1}^{(1)}\left(k_{1} r\right) P_{1}(\mu)+\sum_{l=3 \bmod 2}^{L} c_{l} \mathrm{k}_{l}\left(k_{l} r\right) P_{l}(\mu) & \text { if } r>R \\ c_{0} j_{0}\left(k_{0} r\right)+\sum_{n=2 \bmod 2}^{N} c_{n} \mathrm{i}_{n}\left(k_{n} r\right) P_{n}(\mu) & \text { if } 0<r<R .\end{cases}$

The functions $h_{\nu}^{(1,2)}$ and $j_{\nu}$ are the spherical Hankel and Bessel functions, and the functions $\mathrm{k}_{\nu}$ and $\mathrm{i}_{\nu}$ are the modified spherical Bessel functions of the first and third kind. The incoming $P$ wave amplitude is normalized to unity. Consider the restricted case of $S$ and $D$ waves inside and $P$ and $F$ waves outside. The amplitudes $c_{0}, c_{2}$ and $c_{1}, c_{3}$ are connected by an $\mathrm{SU}(2)$ matrix, $U$. With the boundary conditions of continuity of wave function amplitude and radial gradient at the hoop radius, the variation implies that $c_{2}=0$ and $c_{3}=0$, giving a diagonal matrix $U{ }^{1}$ Under the variational principle, all partial waves except $S$ and $P$ may be neglected. The continuity conditions uniquely determine the phase shift for a given energy, and the peak of the resonance is located at approximately $1.3 \%$ above threshold (see Figure 2). At this energy, the ratio of frequencies is $\nu_{R} / \nu_{T} \approx 2.8$. The lifetime of the resonance is $\tau=\hbar / \Delta E$, where $\Delta E$ is the full width at half maximum of the resonance response $\sin ^{2}(\delta)$. In the present case, a wave function with only $S$ and $P$ waves has an expected lifetime of $\tau \approx 143 m R^{2} / \hbar$, almost two orders of magnitude larger than the characteristic rotation time for the hoop with unit spin.

\footnotetext{
${ }^{1}$ Of course the variational conditions also could be satisfied, for example, by setting $c_{0}$ and $c_{3}$ to zero, but this obviously is a disfavored alternative for energies in the vicinity of the resonance. For incident energies near the $D$ wave threshold, interchanging the roles of $c_{0}$ and $c_{2}$ might make sense, in which case at some intermediate energy there would have to be a transition between the two. We do not study this issue further here.
} 


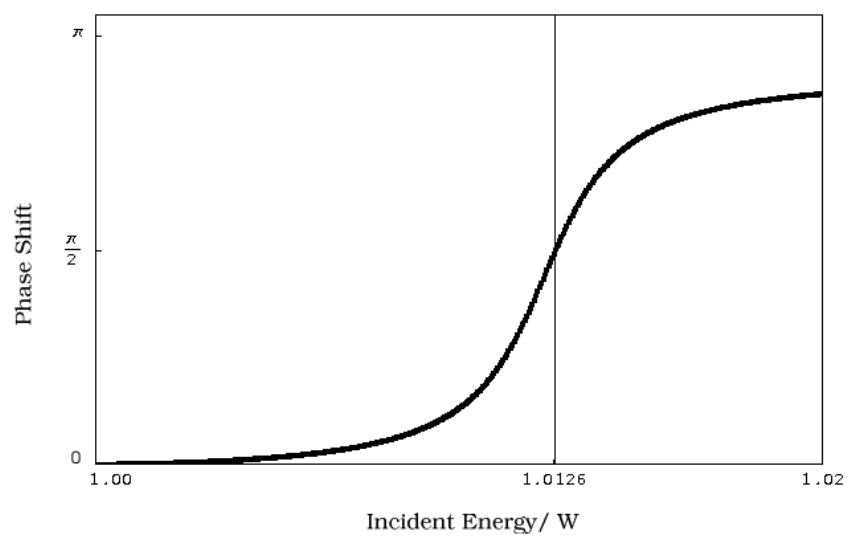

Figure 2: Phase shift versus energy for pure $S / P$ wave

\subsection{High Energy Asymptotics}

A curious feature of the purely $S$ and $P$ interaction is the persistence of an odd phase shift in the high energy limit. In this limit, the validity of neglecting the now classically allowed partial waves of high angular momentum is suspect. The object of this section is to demonstrate that nevertheless the $S / P$ coupling indeed controls the interaction at high energies, so that the result for the asymptotic phase shift is both reliable and understandable. By giving the hoop a definite initial spin state $(l=1)$, we restrict our attention to an incoming wave composed entirely of the $P$ state, while the outgoing wave is an arbitrary combination of odd partial waves. In particular, we are interested in the elasticity of the scattering - the fraction of the probability flux that is reflected back into the outgoing $P$ wave, $j_{1}^{\text {out }} / j_{1}^{i n}$. In the high energy limit, where the transit frequency is much greater than the rotational frequency, the static hoop matching conditions are accurate. The connection rules are well-posed when the number of open channels inside equals the number of open channels outside, that is, $\frac{N+2}{2}=\frac{L+1}{2}$. Setting the amplitude of the incoming $P$ wave to unity, the logarithmic derivatives of the $(N+2) / 2$ interior waves provide a set of linear conditions to specify uniquely the complex amplitude, $c_{l}$, of each outgoing wave ( $l=$ odd integer):.

$$
\frac{j_{n}^{\prime}\left(k_{n} R\right)}{j_{n}\left(k_{n} R\right)}=\frac{h_{1}^{\prime(2)}\left(k_{1} R\right) O_{n, 1}+\sum_{l=1 \bmod 2}^{L} c_{l} h_{l}^{\prime(1)}\left(k_{l} R\right) O_{n, l}}{h_{1}^{(2)}\left(k_{1} R\right) O_{n, 1}+\sum_{l=1 \text { mod } 2}^{L} c_{l} h_{l}^{(1)}\left(k_{R}\right) O_{n, l}} \quad n=\left(0,2, \ldots \frac{N}{2}+1\right)
$$

Primes denote differentiation with respect to the radial coordinate. The factors $O_{n, l}$ are the overlap of Legendre polynomials of degree $n$ and $l$ on the interval $\mu:[0,1]$ 
and arise from the projection of the $l^{\text {th }}$ exterior wave onto the $n^{\text {th }}$ interior wave.

The phase shift $\delta$ in the $P$ wave is defined by $S=c_{1}=e^{i 2 \delta} e^{-\chi}$, where $\chi$ characterizes the inelasticity. Regardless of the choice of truncation (choice of $L$ ), the $P$ wave S-matrix element converges uniformly to $S=-1$ at high energy, which indicates entirely elastic scattering as well as the prevalence of the $S / P$ coupling. We recognize that expanding into an incomplete basis does not insure unitarity, but the lack of inelasticity implies the conservation of probability flux in the limit $L \rightarrow \infty$.

The unique solution to the system of equations (17) as $k_{n} R \rightarrow \infty$ with $k_{n} R \gg n$ is $c_{1} \rightarrow-1$ and $c_{l} \rightarrow 0$ for $l>1$, which is seen upon substitution of the asymptotic behavior of the Bessel and Hankel functions:

$$
\begin{aligned}
-k_{n} \frac{\sin \left[k_{n} R-\frac{\pi}{2}(n+1)\right]}{\cos \left[k_{n} R-\frac{\pi}{2}(n+1)\right]} & =k_{1} \frac{e^{-i(x-\pi / 2)} O_{n, 1}-e^{i(x-\pi / 2)} O_{n, 1}}{e^{-i(x-\pi)} O_{n, 1}-e^{i(x-\pi)} O_{n, 1}} \\
k_{n} \tan \left[k_{n} R-\frac{\pi}{2}(n+1)\right] & =k_{1} \tan \left(k_{1} R-\pi / 2\right) .
\end{aligned}
$$

The periodicity of the tangent along with the relation $k_{n}^{2} R^{2}+2 n(n+1)=k_{1}^{2} R^{2}$ between wavenumbers implies that the conditions (17) are satisfied for all interior waves, $n=0$ or $n \in$ even integers, provided $n \ll k_{n} R$. The relative minus sign between the incoming and (equal-magnitude) outgoing waves corresponds to a phase shift of $\pi / 2$ and entirely elastic scattering. We also note that these arguments apply equally well to the phase shift of any odd incoming wave (with $l \ll k_{l} R$ ), for example an $F$ wave. Against our intuition, the $S / P$ coupling is strong even at high energies, while an equipartition of energy would imply current in each outgoing partial wave, possibly in a Boltzmann distribution. That the particle feels the AB effect at high energy may seem surprising at first glance, for typically the limit of high energy is also the limit of high quantum number - where the correspondence with classical physics is realized. In the present model, the incoming wave is spherical, invariably encounters the $\mathrm{AB}$ phase shift (i.e., $2 \delta=\pi$ ) and is reflected primarily into the lowest outgoing wave. We can gain some insight into the elasticity by reasoning from a quasi-classical standpoint. For low angular momentum channels the incident particle has a small impact parameter, $b \sim l / k_{l}$. For collisionless scattering, an abrupt shift to a high impact parameter is suppressed, because the overlap of particle states localized around the interior of the hoop and high angular momentum outgoing channels $(b \gg R)$ is small. To estimate the frequency of collisions, consider an incident wave packet, whose transverse dimension will go as $1 / k$. The interaction will be significant only if the wave packet arrives to an "end-on" hoop. From the hoop, the field of view subtended by the wave packet is roughly $1 / k R$, giving a probability of interaction of $1 / \pi k R$. The expected energy transfer of a given event goes as $p^{2} / k R \sim \hbar p / R$, which vanishes in the limit $\hbar \rightarrow 0$. Indeed, the fractional energy transfer between particle and hoop vanishes as $\hbar / p R$ in the limit $p \rightarrow \infty$. 
Actually the above is an overly conservative estimate of suppression of collisions between particle and hoop. Because we are interested in total angular momentum zero, for an incident wave packet along the $z$ axis the hoop normal should be oriented close to the $z$ axis also. Thus the system of wave packet plus hoop projected to zero total angular momentum must have negligible probability for the particle to collide with the hoop instead of simply going through it.

\section{Constructing a 'Realistic' Model}

Throughout our analysis we have considered an idealized system with a number of key assumptions. We now shall attempt to realize these assumptions in a material system, and give an estimate of the resonance lifetime. In terms of the parameters of the system, the lifetime is $\tau \approx 143 \mathrm{mR}^{2} / \hbar$. As the resonance is narrow and close to threshold, we expect an extremely long-lived state. The chances of finding a measurable lifetime are greater in a system of smaller (micro- to nano-scale) dimensions.

A carbon nanotube might serve as the hoop. The parity reversal across the hoop radius relies on a tuning of the magnetic flux to one half an Aharonov-Bohm flux quantum, which is precisely a superconductor quantum of flux. Although carbon nanotubes may be superconducting, to achieve a "longitudinal" orientation of flux inside the hoop would demand current wrapping around the thinner dimension of the hoop, which has not been demonstrated as far as we are aware. The calculation of the lifetime involved an assumption of infinite mass for the charged particle. Furthermore, interactions between the hoop and particle were assumed to be negligible. Two appropriate ratios that quantify these assumptions are $\alpha \equiv R / r_{p}$ and $\beta \equiv m_{p} / m_{H}$ with $r_{p}$ as the radius of the spherical charged particle, which we'll take as composed of lead. ${ }^{2}$

Let $N$ and $n$ denote the number of carbon atoms required to span the larger and smaller circumference of the hoop, respectively. The total number of carbon atoms goes as the product $N n{ }^{3}$ In terms of the ratios, $N \sim\left(\alpha^{3} \beta n\right)^{1 / 2}$, while $\tau \sim$ $n N^{3} \sim \alpha^{9 / 2} \beta^{3 / 2} n^{5 / 2}$. A plausible choice is $\alpha=50, \beta=50$, and $n=50$, which implies $N \approx 9 \times 10^{4}$ and $\tau \approx 6 \times 10^{5} \mathrm{sec} \approx 10^{2} \mathrm{hr}$. Barriers to further reducing the ratios to shorten the lifetime arise from the need to minimize the collisional or direct interactions between the particle and the material of the hoop, the ultimate rigidity and strength of the hoop, and the conditions for maintaining a superconductor quantum of flux in the hoop - if that is possible at all!

Suppose one actually could make such a system with roughly these parameters.

\footnotetext{
${ }^{2}$ Physical constants: The mass of a carbon atom is $m_{c} \approx 2 \times 10^{-25} \mathrm{~kg}$, the length of a carboncarbon bond is $l_{c} \approx 1.5 \AA$, and the bulk density of lead is $\rho \approx 1.1 \times 10^{4} \mathrm{~kg} / \mathrm{m}^{3}$

${ }^{3}$ We are assuming a single layer of carbon atoms constitutes the surface of the tube
} 
To avoid overwhelming gravitational stresses, observations would have to be done in a freely falling laboratory. While this is conceivable, another constraint is not (at least in the foreseeable future): To detect such a long lifetime, one must work at a temperature low enough so that thermal excitations would not free the hoop from the particle in a time short compared to the resonance lifetime. Conservatively, this means a temperature less than $\mathcal{O}\left(\hbar / m R^{2}\right) \approx 10^{-14} \mathrm{~K}$. That is a very long way from the regime of low temperatures currently attainable in the laboratory.

While we are trying to make our considerations realistic, we should return to an earlier assertion that the spin $s_{z}$ of the hoop about its symmetry axis must vanish. For a maximally symmetric hoop, indistinguishable atoms would be interchanged under a rotation by the angle $2 \pi / N$, implying if the atoms are spinless bosons (as is true for ${ }^{12} \mathrm{C}$ ) the relation $e^{2 \pi i s_{z} / N}=1$, or $s_{z}$ is an integer multiple of $N$. Evidently for unit multiple this would lead to an energy $\sim N^{2}$ times greater than for rotation of the $z$ direction, and at low temperatures this kind of excitation would be literally frozen out. Of course, in practice there would be imperfections in the rotational symmetry of the hoop about its axis, resulting from the presence of impurity atoms. Because the kinetic energy of such an atom involves a moment of inertia smaller than that of the hoop as a whole by $\sim N n$, exciting this degree of freedom would involve energies larger than for rotation of the symmetry axis by this factor, again negligible at low temperatures. There also could be excitations corresponding to transverse or longitudinal sound waves traveling along the hoop. These would have energy larger than for the axis rotations by $\sim M R v / \hbar$, where $v$ is the speed of such waves - again clearly suppressed.

\section{Conclusions}

We have seen that the effective attraction of 'electron' and flux hoop is insufficient to produce binding, because localizing the particle within a radial dimension of roughly $r=R$ costs slightly too much energy, compared to that required for release of the particle in a $P$ wave, the allowed external configuration of lowest energy. Because the attraction is optimized by making the electron much more massive than the hoop, the binding is more like a light wire cage surrounding an elephant-i.e., the hoop is attracted to the electron rather than vice versa. Once again, as in examples mentioned in the introduction, the quantum attraction actually is a side effect of positive or repulsive uncertainty-principle energy: Surrounding by a wall is a method of trapping just as effective as digging a hole. Still, examples of such quantum walls are few compared to the myriad illustrations of net quantum repulsion, going back all the way to the stabilization of ordinary matter against collapse, which results from a combination of Heisenberg uncertainty-principle energy with the Pauli exclusion 
principle [11].

Although the attraction of hoop to charge is insufficient for binding, it still gives rise to a resonance just above threshold for scattering in the $P$ wave. In this neighborhood of energy, the rotation time of the hoop is less than the transit time, and much less than the mean residence time of the particle. We do not know a simple approximation to the exact boundary conditions. High angular momentum channels are classically forbidden, and so should be weakly coupled to the incoming wave. We believe the motion involves predominantly the $S$ wave (inside) and the $P$ wave (outside). The S-matrix version of the Kohn variational principle supports this view. Conversely, there also should be a resonance in total spin zero $S$ wave scattering, at an energy in the vicinity of each odd (inside) wave threshold.

In some sense, the $\mathrm{AB}$ effect may be considered as a two dimensional phenomenon, because the two dimensional problem solved exactly by Aharonov and Bohm captures fundamental features of the effect. Still, as our world has three space dimensions, it is interesting to examine the nature of a finite $\mathrm{AB}$ system in that world. However, we've seen that the degree of difficulty in theoretical description increases with the number of dimensions, so that what was completely soluble in two dimensions already becomes quite challenging in three, only partially solved here with the help of maximal rotational symmetry. The uniqueness of the transition conditions at the hoop radius $R$, specifically the parity reversal, is responsible for the unusual characteristics of the system, including an attractive interaction in the configuration with odd partial waves outside, as well as the asymptotic value $\pi / 2$ for the phase shift at high energy.

If the theoretical difficulty of this system is significant, the difficulty of studying it in the laboratory may be overwhelming. In that case, perhaps we may content ourselves with the theoretical discussion as an exotic illustration of some basic principles, and hope that this example may lead to others that are more amenable to experimental study.

ASG thanks Sidney Coleman for provocative discussions about this system more than a decade ago, Yakir Aharonov, Shmuel Nussinov, and Sandu Popescu for background on other systems with quantum attraction, Walter Kohn for drawing attention to Ref. 9, the National Science Foundation and the Department of Energy for partial support, and the Stanford Linear Accelerator Center Theoretical Physics Group for hospitality during a sabbatical leave. Early stages of this work were carried out under the Research Experiences for Undergraduates program of the National Science Foundation at the Stony Brook University Physics and Astronomy Department. The support mentioned does not imply any warrant by the supporting agencies for the results obtained. 


\section{References}

[1] Y. Aharonov and D. Bohm, Phys. Rev. 115, 485 (1959)

[2] T. T. Wu and C. N. Yang, Phys. Rev. D 12, 3845 (1975)

[3] M. V. Berry, Proc. Roy. Soc. London 392, 45 (1984)

[4] B. Simon, Phys. Rev. Lett. 51, 2167 (1983)

[5] Y. Aharonov and J. Anandan, Phys. Rev. Lett. 58, 1593 (1987)

[6] R. L. Schult, D. G. Ravenhall, and H. W. Wyld, Phys. Rev. B39, 5476 (1989)

[7] P. Exner and P. Seba, J. Math. Phy. 30, 2574 (1989)

[8] J. Goldstone and R. L. Jaffe, Phys. Rev. B45, 14100 (1992)

[9] J. Z. H. Zhang, S. I. Chu, and W. H. Miller, J. Chem. Phys. 88, 6233 (1988)

[10] W. Kohn, Phys. Rev. 74, 1763 (1948)

[11] J.L. Lebowitz and E.H. Lieb, Phys. Rev. Lett. 22, 631 (1969) 